\title{
Laparoscopic Radical Prostatectomy
}

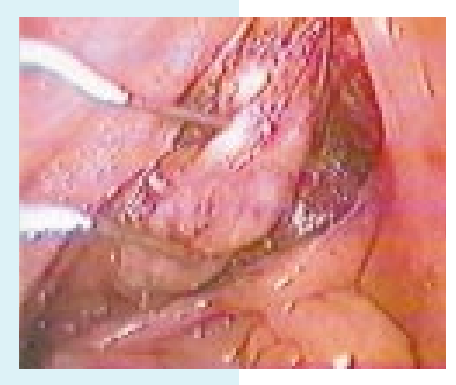

Bertrand Guillonneau, M.D.

Arnon Krongrad, M.D.

Guy Vallancien, M.D.

With a COMMENT bY

Victor A. Politano, M.D. 
C O M M E N T

January 19, 2000

The $20^{\text {th }}$ century brought about many exciting changes in the field of medicine. Anesthesia, antibiotics, blood replacement, better imaging and blood testing, the unraveling of DNA and identification of genes have given us the ability to diagnose early and treat more effectively. Patients with urologic disease have benefited from all of these advances. The century now starting is expected to be even more exciting.

In a world of rapid innovation, we honor the firstrule of medicine: primum, no nocere. We sedk lessmorbid ways for accomplishing surgical goals. Laparoscopy offersusa way to lessmorbid surgery. As hardwareand softwareadvanœ, the urologist'sability to offer his patientlessmorbid operations will belimited only by ability to adoptthenewtechniques. Even manual dexterity, a determinant of surgical outcome, will diminish in importanceas robotsincreasingly collaborate with human surgeons.

We saw the evolution of open stone surgery to endoscopic stone surgery. Today, we see the evolution of open organ ablation and reconstruction to laparoscopic organ ablation and reconstruction. For the urologist of the future, mastery of laparoscopic surgery will be imperative.

The $20^{\text {th }}$ century opened with the seminal work in Paris of Albarran, Motz, and Perearneau who gave us an anatomic foundation for the prostate surgery that was to come. The $21^{\text {st }}$ century is opening with work of comparable meaning: the first realistic laparoscopic operation for prostate cancer. Like the foundation laid 100 years ago by our urological forebearers, the foundation presented here heralds numerous innovations to come.

Drs. Guillonneau, Krongrad, and Vallancien areamong today's most thoughtful urological innovators. As presented in vivid simplicity, clarity, and precision, their monograph on laparoscopic radical prostatectomy embodies the finest attributes of our profession: surgical innovation, technical innovation, collaboration, and the commitment to disseminate knowledge.

I applaud the authors on a job well done. I wish our patients and us a century of innovation as exciting as the last.

\section{Wectore tr. Pletexo}

Victor A. Politano

Former President, American Urological Association

Miami, Florida 


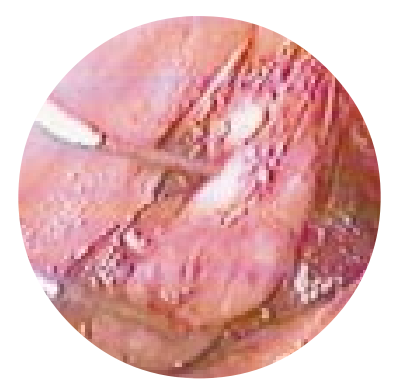

\section{N T R O D U C T I O N}

Laparoscopic radical prostatectomy was first described in an abstract in $1992 .{ }^{1}$ At that time, only two cases had been completed and formation of the vesicourethral anastomosis was exceedingly time-consuming. Indeed, equipment for suturing in the early 1990s was rather crude and the needle holders commonly did not firmly grip the needle. Likewise, experience in intracorporeal and extracorporeal knotting was still in its earliest stages. From 1992 to 1996, no information was published on laparoscopic radical prostatectomy.

In 1996, Priceetal. reportedlaparoscopic radical prostatectomyin thedogmodel. ${ }^{2}$ Among six dogs, four had a good outcome. Also in 1996, Raboy etal. completed a clinical case of extraperitoneal radical prostatectomy; negative margins and excellent continence were reported ${ }^{3}$ In 1997, Schuessler et al. formally published their early experience. ${ }^{4}$ To this point, no one had published a clinical series.

Despite faltening steps and obstacles early in its development, today the laparoscopic radical prostatectomyisin dinical useand totally standardized; theworld dinical experienceisestimated to exceed 500 cases. Clinical outcomes are being published and appear to be excellent. ${ }^{5}$

The advantages of the laparoscopic approach include improved visualization of the anatomy, reduced blood loss, better preserved anatomical structures, and shorter convalescence. The laparoscope, given its small size and maneuverability, can provide the surgeon with hitherto unobtainable anatomical images.

Today's laparoscopic radical prostatectomy is feasible, reproducible, standardized, and can betaught. The specific technique described here, developed over two years in clinical practice at the Institut Montsouris in Paris, is a conceptual hybrid of well-known open surgical techniques. Forinstance, in itsapproach to theprostatic pedicles, the Montsouris technique resembles the antegrade Campbell technique; in its approach to the dorsal venous complex and neurovascular bundles, the laparoscopic approach resembles the retrograde Walsh technique.

While it poses a real technological learning curve, there is nothing magic about this operation and the technical points are well known. A surgeon familiar with prostate anatomy $y^{6}$ and laparoscopic techniques should, after watching a few cases, be able to learn the laparoscopic radical prostatectomy.

\section{TECH I Q U E}

1. Patient selection and preparation:

Patient selection is essentially the same as for open radical prostatectomy. As with open surgery, previous abdominal and/or perineal surgery, radiation, and/or androgen deprivation may complicate organ dissection.

Since operations are done under general anesthesia, patients should receive nothing by mouth for at least six hours before surgery. No bowel preparation is given. A single intravenous dose of a 3rdgeneration cephalosporin and 2,500 units of low molecular weight subcutaneous heparin are given on call to the operating room. Patients admitted the day before surgery receive 3,500 units of low molecularweight heparin the night before surgery.

\section{Patient position:}

Thepatientissupinewith armsathissides, with soft shoulder supports (Figure 1). The legs are parted on spreader bars to provide access to the urethra andanus; no O'Connor drapeisused norisa small roll placed under the lumbar spine. The abdomen is shaved from the costal margins to the pubic bone. The abdomen, penis, scrotum, upper thighs, and perianal region are prepared 


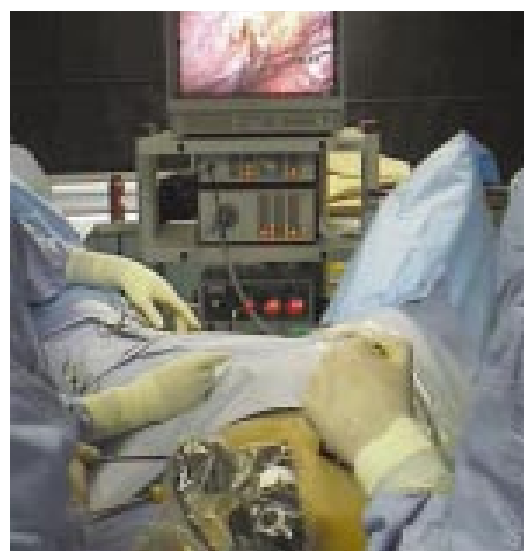

Figure 1. Patient positioning: The patient is supine, in steep Trendelenburg position, with legs parted. The urethral meatus and anus are exposed, prepped, and draped.

with iodine-based disinfectant and draped; the legs are draped individually. The table is set in extreme Trendelenburg position, with the monitor between the patient's legs. A "leaning post" is constructed for surgeon comfort from a foam-wrapped horizontal table attachment. A urethral catheter is inserted to drain the bladder.

\section{Surgeon, trocar, and robot position:}

A right-handed surgeon stands on the patient's left, a left-handed surgeon on the right. An assistant stands opposite the surgeon, provides exposure, and works the suction device.

An incision is made along the inferior crease of the umbilicus. A needle is introduced into the abdominal cavity, which is inflated with $\mathrm{CO}_{2}$ to a pressure of $15 \mathrm{~mm} \mathrm{Hg}$. The needle is removed and a 10-mm trocar is inserted (Figure 2). The laparoscope is inserted and used to transilluminate the abdominal wall before subsequent trocars are placed. A second $10-\mathrm{mm}$ trocar is placed at

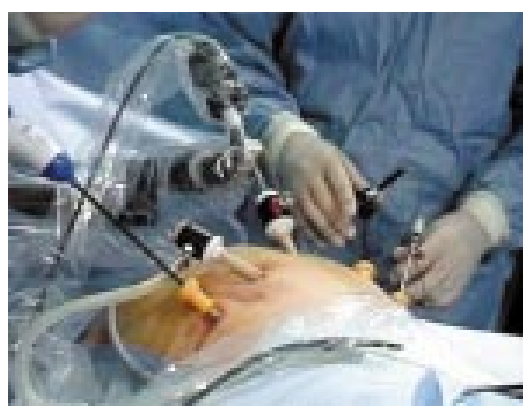

Figure 2. Surgeon, trocar, and robot position: The laparoscope is held by a voice-activated robot, while the surgeons trade instruments and hand position. A right-handed surgeon stands on the left side of the table. A suction device, operated by the assistant, is seen in a right-sided trocar. The surgeon typically works through the two left ports and/or the more medial right-sided port.

McBurney's point, to which the $\mathrm{CO}_{2}$ line is then connected (placing the gas line on a trocar other than that used for the lens minimizes lens fogging); this trocar is later used for retrieving the specimen. A 5-mm trocar is placed inferolateral to the second. A 5-mm trocar is inserted in the left iliac fossa, and another $5-\mathrm{mm}$ trocar is placed inferolateral to it. Alternatively, the last trocar is placed in the midline half-way between the umbilicus and the pubis.

The abdomen is inspected for trocar injury, bleeding, metastases, and adhesions. Anatomical landmarks to observe are: obliterated urachus (median umbilical ligament) in the midline, the Foley balloon and tip in the bladder, the two obliterated umbilical ligaments (medial umbilical ligaments), and the vasa deferentia. If the operation is to include a pelvic lymphadenectomy, this is done first in the standard laparoscopic fashion. The lymphadenectomy peritoneal incisions are not closed, nor are they used for the prostatectomy.

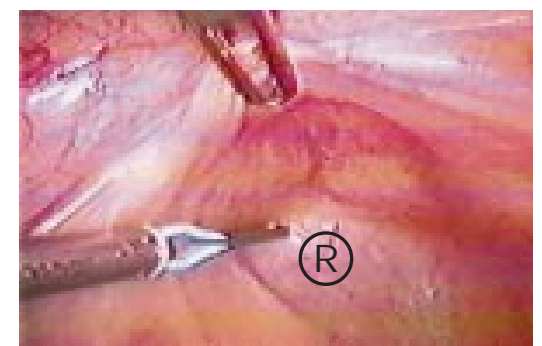

Figure 3. Peritoneal arches: A forceps elevates the superior peritoneal arch, representing the course of the ureters. This arch should be avoided. Another forceps points to the inferior peritoneal arch, representing the peritoneal fold along the rectum $(R)$. Behind this fold is the confluence of the vasa and seminal vesicles.

Thelaparoscopeis connected magnetically to a voice-controlled robot whose base connects to and moves with the table. The robotis directed only by the surgeon's voice.

\section{Freeing the seminal vesicles:}

One finds the vasa and seminal vesicles at the inferior-most peritoneal reflection, posterior to the bladder and anterior to the rectum. The assistant gently retracts the rectum superiorly, which can be easily identified by movement with a bougie inserted through theanus. The surgeon can note the appearance of two peritoneal arches (Figure 3).

The Foley tip generally lies above the two arches. The superior arch represents the approximate location of the ureters and should be avoided. The inferior arch, at the depths of the peritoneal reflection, is created by the coming together of the vasa in the midline, with the seminal vesicles lying just lateral.

The surgeon incises the peritoneum at the level of the second, inferior peritoneal arch to find the vas (Figure 4), which may be 


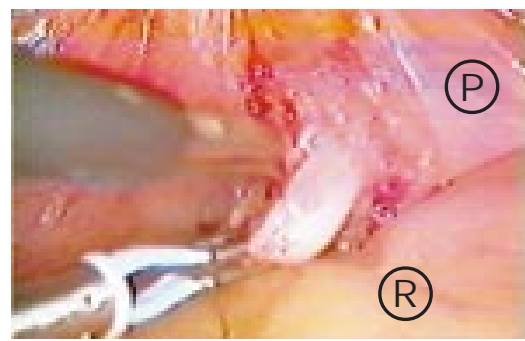

Figure 4. Exposed vas deferens: The peritoneum $(P)$ is incised to allow access to the vas deferens, which may be retracted, coagulated, and transected. This portion of the vas overlies the middle of the seminal vesicle. $R=$ rectum.

cauterized with the bipolar forceps and transected. The vas artery runs along the vas, between the vas and the seminal vesicle. This artery must be coagulated.

Once the vas is transected, the inferior portion may be traced to expose the seminal vesicle. The seminal vesicle is dissected and left attached only at its base (Figure 5), with care to cauterize its two medially directed arteries, one at the tip and another at the base. The seminal vesicles must be well mobilized before the prostate dissection.

\section{Opening Denonvillier's fascia:}

Incision of Denonvillier's fascia allows an easier dissection of the prostatic pedicles later in theoperation, after the bladderneck isincised. While the surgeon is working, the assistant should retract the seminal vesicles and vasa anteriorly, which places Denonvillier's fascia on some tension.

The Denonvillier's fascia will appear to have sagittal (cephalo-caudal) striations under the magnification of the laparoscope

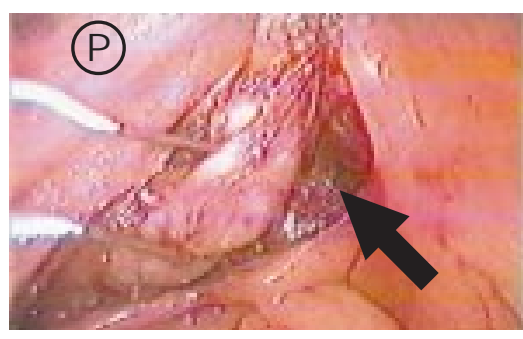

Figure 5. Exposed seminal vesicle: After the vas is transected, the seminal vesicle $(P)$. The base of the seminal vesicle is left attached and the rest is freed. Often two arteries can be seen and coagulated. Denonvillier's fascia (arrow) is visible. is dissected through the incised peritoneum

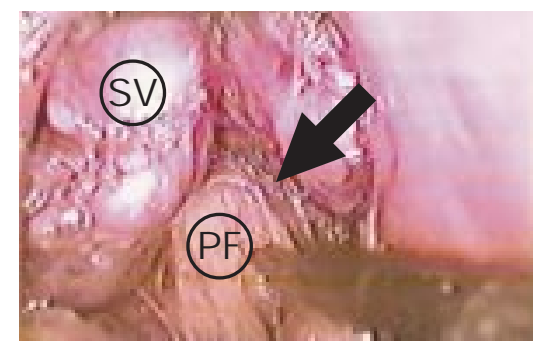

Figure 6. Denonvillier's fascia: With the rectal bougie directed posteriorly and with both seminal vesicles (SV) freed and retracted anteriorly, the saggital striations of Denonvillier's fascia become apparent (arrow). Once Denonvillier's fascia is incised, the surgeon sees yellow prerectal fat (PF). Exposure of the prerectal fat introduces the surgeon to a safe plane of dissection that extends under the prostate to the rectourethralis.

(arrow, Figure 6). The surgeon should incise these striations longitudinally, close to the ampulla of the vas and the seminal vesicles. As soon as a shallow incision is made in Denonvillier's fascia prerectal fat is seen, which provides a safe plane of dissection inferiorly to the posterior surface of the prostate; blunt dissection behind the prostate at this stage will make dissection of the pedicles simpler later. The limit of the dissection at this point is the rectourethralis.

To assist in general orientation, it is often useful to withdraw thelens and geta global view the operating field (Figure 7). Orientation is helped by theplacementand movement of a rectal bougie.

\section{Dropping the bladder:}

The bladder is filled with approximately 120 cc. of saline to help identify the contours and pull it posteriorly (Figure 8). Bladder dissection starts with an incision lateral to the bladder contour but medial to the medial umbilical ligament; if a lymphadenectomy was done, fresh incisions are made medial to the medial umbilical ligament. This incision is carried down to the vas and up to the abdominal wall. Dissection is continued anteriorly and caudally until the pubic bone is contacted. It is unnecessary to divide the medial umbilical ligaments. By staying close to the medial aspect of the medial umbilical ligaments and heading laterally and caudally from there, the pubic ramii are encountered; the chance of incising the bladder is thereby minimized.

Next, the urachus is divided in the midline and the dissection is carried down to the level of the symphysis pubis, traversing the light, prepubic areolar tissue of the space of Retzius ( Figure 9). Again, in order to avoid any bladder injury, the dissection of the urachus should be as high as reasonable. This dissection is done with bipolar or monopolar electrosurgical current.

Thelaparoscopic dissection mimicsan open dissection of the bladder and aims to cause the bladder to fall posteriorly to allowaccess 


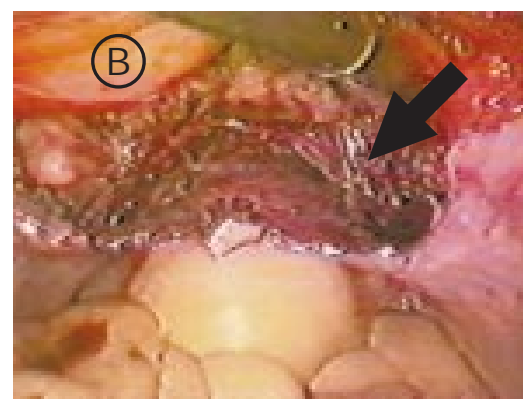

Figure 7. Global view: Scissors tent up the bladder (B), push away the right seminal vesicle, and reveal Denonvillier's Fascia (arrow). The peritoneum is draped over the rectum, outlined as a round organ by a large bougie.

to the space of Retzius. It is also important to free the bladder well from its anterior attachmentsin order to permita tension-free vesico-urethral anastomosisattheend of the operation. After the bladder is freed anterior and lateral, it is then emptied with a syringe: because the patient is in exaggerated Trendelenburg position, spontaneous emptying is never complete.

\section{Approaching the dorsal vein:}

Once the bladder is dropped, the superficial dorsal vein coursing over the prostate should be coagulated. This vein is then incised and the fat over the fascia of Zuckerkandl covering the prostate can be resected or swept cephalad and laterally. In most cases, the endopelvic fascia has a moon-shaped gap - the "gap of Guy" a natural opening that may be entered to permit access to the periprostatic space. The endopelvic fascia isincised on its line of reflection, allowing the approach of the levator ani muscles arcing laterally. Toward the puboprostatic ligaments, one vein is often found that must be coagulated and transected. In some cases, one may

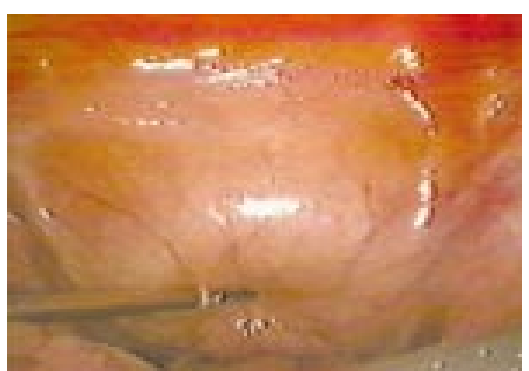

Figure 8. Bladder contours: A forceps indents the filled bladder. Dissection is started along the lateral aspects of the bladder.

observe the "continence" nerves described by Steiner. ${ }^{7}$

Incision of the puboprostatic ligaments is done to facilitate dissection of the dorsal venous complex, performed with a curved scissors, using light current (Figure 10).

\section{Ligating the dorsal venous complex:}

The tiny veins around the puboprostatic ligaments may be safely cauterized with a bipolar forceps. The dorsal venous complex can then be ligated with a 2-0 resorbable suture passed with a \#36 needlefrom one side of the complex to theother. Afigure of-8may be placed or two separate sutures can be placed, at the surgeon's discretion and depending on the breadth of the plexus, and thesuturetied. Abackbleeding stitch can also be placed on the prostate at the surgeon's discretion. This suturecan be helpful during thesubsequent dissection atthebladderneck, because a locking grasping forceps can be affixed to the tags emanating from the knot on the suture, thereby providing the surgeon with the means to place some upward traction on the prostate.

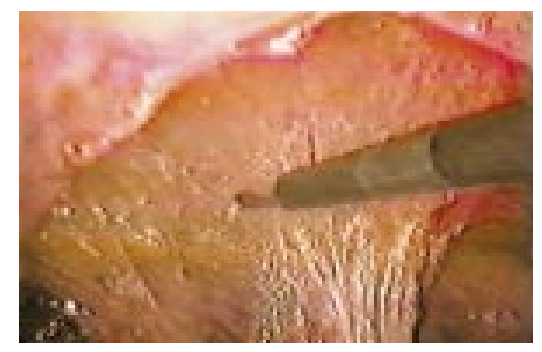

Figure 9. The space of Retzius: The areolar tissue appears yellow and reflects light. This tissue is traversed to reveal first the pubic bones and then the endopelvic fascia.
In ligating the dorsal venous complex, it will help the right-handed surgeon to pass the needle from the right to the left side via the right-sided port. The needle should be situated such that the curve of the needle follows the curve of the symphysis pubis (Figure 11). The dorsal venous complex may be transected later.

\section{Dissecting the bladder neck:}

The plane between the prostate and bladder neck is often elusive in open surgery, and so it is in laparoscopic surgery. To identify the bladder neck, the anterior prevesical fat is retracted superiorly, causing a faint outline of the prostatovesical plane. As with open surgery, this plane is developed with sharp and blunt dissection. The bipolar forceps are useful in controlling the minor bleeding encountered.

The surgeon identifies the urethra, incising the anterior wall to expose the Foley catheter. The catheter balloon is then deflated, and the catheter is pulled up and into the abdomen (Figure 12) to expose 


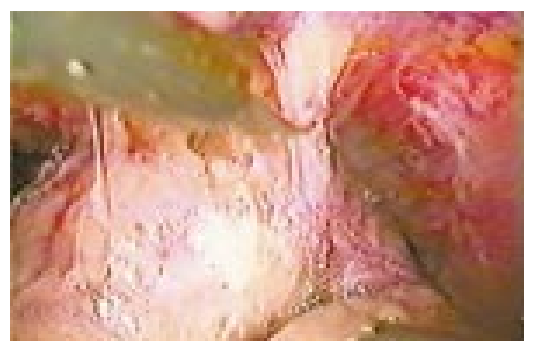

Figure 10. Puboprostatic ligaments: In the laparoscopic view, the puboprostatic ligaments appear massive and are easily incised. Small veins coursing in the surgical field may be coagulated with bipolar current.

the lateral and posterior urethral walls, which are incised. The Foley catheter is removed, and the assistant exposes the posterior face of the bladder neck holding the posterior bladder mucosal incision. With care, the bladder neck is well preserved and the ureteral orifices lie far from the region of dissection.

As one proceeds to incise the junction between the prostate and bladder, it is important to direct the dissection straight posteriorly. Dissecting the prostate from the bladder does not present the urologist with a distinct or easily developed plane; rather, one must methodically go through somewhat vague planes in this area. Excessive bleeding or recognition of muscle type tissue indicates that one is getting too close to the bladder or is into the detrusor of the bladder. By the same token, development of a "natural" plane that can be easily entered can be a "fool's path," asit will bring one into the prostate itself (adenomectomy plane). With meticulous dissection straight posterior, the posterior bladder neck is encountered and incised, allowing access

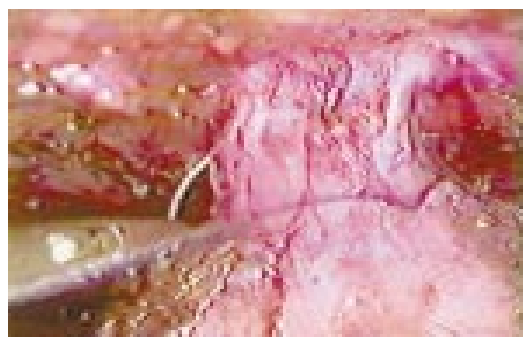

Figure 11. Stitching the dorsal vein: The venous complex of Santorini is ligated with a 2-0 resorbable suture. Incision of the venous complex is delayed until after the completion of the incision of the prostatic pedicles.

to the previously dissected retrovesical space, after transversely incising a final fascia, the "anterior layer" of the Denonvilliers fascia.

The prostatic pedicles becomeapparent at this point because they have been completely dissected on all sides. As one completes incising the posterior bladder neck, the bladder neck retracts naturally out of view, but is easily retrieved with slight traction on the bladder.

\section{Incising the prostatic pedicles:}

Unlike in retrograde retropubic radical prostatectomy, in the antegrade laparoscopic operation, the pedicles are laid virtually bare before they are incised. The vascular prostatic pedicles are separated by a thin fat plane from the postero-lateral neurovascular bundles. To best expose them, the surgeon grasps the vas and seminal vesicle through the space between the prostate and the posterior bladder neck and pulls them up to expose the pedicle to be incised (arrow; Figure 13).

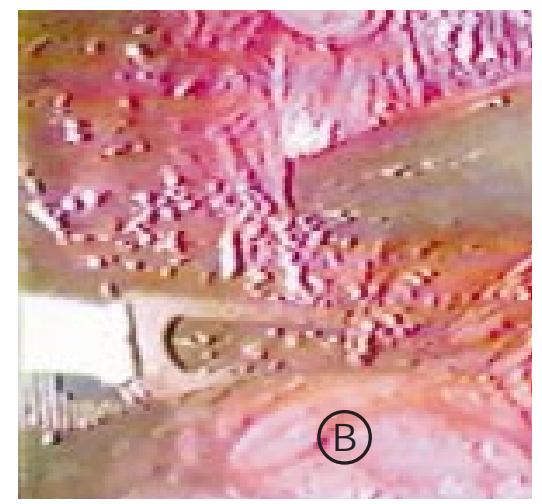

Figure 12. Opening the bladder neck: An anterior urethrotomy has been made with subsequent retrieval of the Foley catheter. The Foley catheter is pulled up as the bladder $(B)$ is retracted. The suction tip is on the posterior bladder neck, and the next step will be to incise the posterior wall bladder neck mucosa.

The pedicle is characterized by wellvisualized arteries. To be efficient in this operation requires systematic bipolar coagulation of the arteries. Because of the dissection and traction on the vas and seminal vesicle, the arteries appear to be rising vertically, which is a distortion of their natural orientation. Under the magnification of the laparoscope, the prostatic arteries, seen in perfect 5 and 7 o'clock position as described by classical anatomists, ${ }^{6}$ appear sizable. The pedicle arteriesmayall be cauterized with bipolar current; metal clips are unnecessary and crude. The surgeon reorients himself periodically during the dissection of the pedicles to keep in mind the location of the postero-lateral neurovascular bundles.

11.Preserving the neurovascular bundles:

Atter transection of the pedicles, the surgeon finds a flimsy tube of fat, nerves, and vessels that runs along the posterolateral surface of the prostate, from the base to its insertion near the urethra into the pelvic muscular floor. This structure contains 


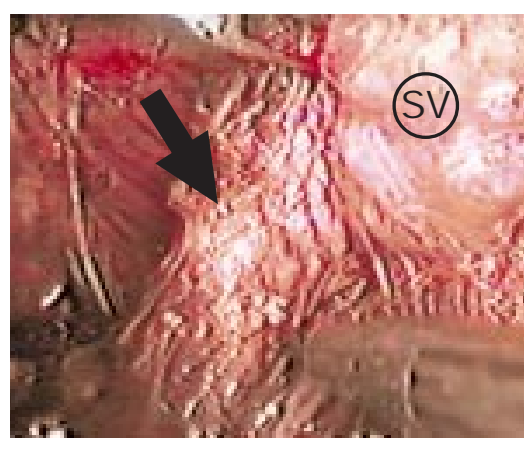

Figure 13. Incising the prostatic pedicle: The seminal vesicles are brought into the operating field through the incised bladder neck. Here, the left seminal vesicle (SV) is pulled to the right, placing the left pedicle (arrow) on tension. The vessels in the pedicle can be individually identified and coagulated close to the prostate, with care to preserve the neurovascular bundles that lie beneath them (Figure 14).

the neurovascular bundles (arrow; Figure 14). To preserve the bundles, it is necessary to make a lateral incision in a thin, visceral fascia that laterally covers the peri-bundle fat. This incision exposes laterally the bundles and capsular arteries, which rise vertically into the prostate. Theses small arteries must be coagulated with thin forceps to diminish the risk of thermal injury. There are more arteries backward, near the prostatic pedicle, than forward, near the apex, where the bundle must be dissected off the prostate with blunt and sharp dissection. Hemorrhage around the bundles is always minor and, for the sake of neurovascular integrity, should be neglected. The neurovascular bundles must be dissected from the base of the prostate to the entrance into the pelvic muscular floor, posterolateral to the urethra.

\section{Incising the urethra:}

The mechanics of the laparoscopic urethral dissection are consistent with

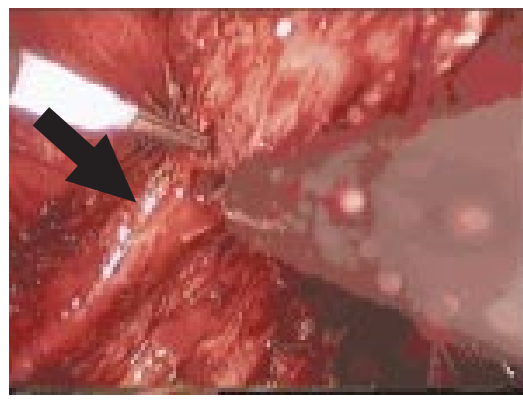

Figure 14: Preserving the neurovascular bundles: Once the pedicle has been transected, the surgeon encounters a tubular structure composed of fat and the neurovascular bundle (arrow). This structure should be carefully dissected from the prostate, from its base to its insertion in the pelvic floor. Coagulation should be minimal, to spare nerve integrity.

those of continence-sparing open radical prostatectomy, ${ }^{7}$ which is designed to preserve the pelvic nerve and intrapelvic branches of the pudendal nerve. In some cases, these nerves can be seen coursing along the levator ani; none of us can remember having seen these nerves in open surgery.

Because the dorsal vein has been ligated and the pedicles have been incised, there is little bleeding when the dorsal vein complex is incised. This incision is made by first coagulating with bipolar forceps and then incising the complex. The dorsal vein complex is retracted anteriorly to expose the anterior urethral wall. The urethral wall is then incised with a cold knife, and the urethral sound is pushed through the anterior urethrotomy and into the pelvis ( Figure 15). The back wall of the urethra is now similarly incised with the laparoscopic knife or scissors.

Gentle traction isapplied to pull the prostate superiorly. If a backbleeding suture was placed on the prostate, it may now be grasped and retracted. The dissection is

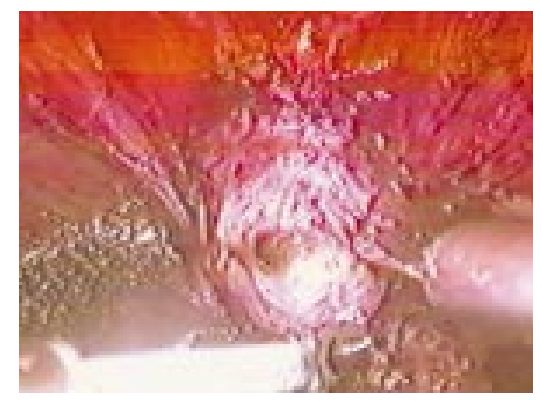

Figure 15. Incising the urethra: After the prostatic pedicles are incised, the previously ligated dorsal venous complex (Figure 11) is incised. The urethra is then revealed, and its anterior wall is incised with a cold knife, as shown here. A metal sound (S) may be inserted into the urethra to help identify the anatomy.

continued close to the prostate. The rectourethralis appears rather attenuated with this approach and represents the final attachment of the prostate. Division of the rectourethralis close to the prostate completely frees the specimen (Figure 16), which is temporarily placed in a paracolic gutter.

\section{Forming the urethero-vesical} anastomosis:

It is not necessary to evert the bladder mucosa, nor is it necessary to narrow the bladder neck. Knots may beformed in or out of the anastomotic lumen. Throughout this portion of the procedure the surgeon works with two needle holders.

The anastomosis is made with interrupted stitches; running stitches have been tried and abandoned, because they are more time consuming and offer no clinical advantage over interrupted stitches.

The anastomosis is well constructed with 3-0 resorbable suture; a \#26 needle works well with the laparoscopic approach, and 


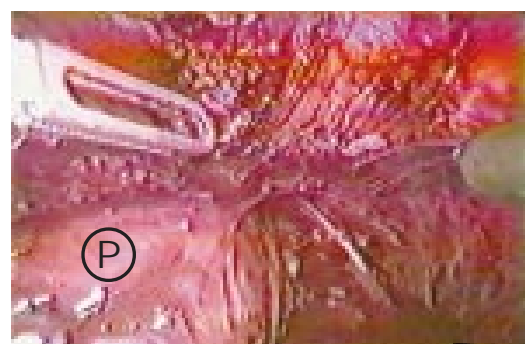

Figure 16. Freeing the specimen: The prostate $(P)$ is retracted to the left, revealing a few remaining attachments

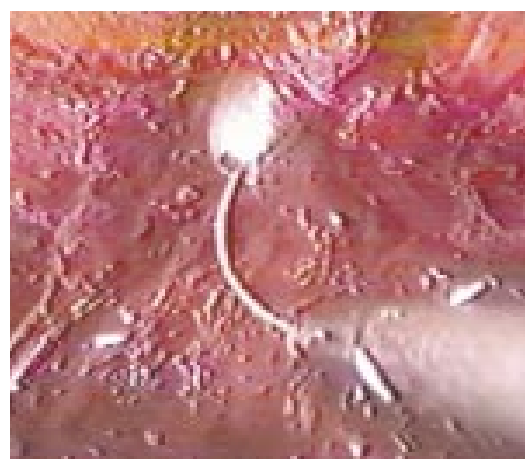

Figure 17. Vesico-urethral anastomosis: With the specimen stored temporarily in a para-colic gutter, the anastomosis is constructed. Here, a 2-0 resorbable stitch is guided into the urethral lumen by a urethral sound with a depressed tip. all ties are thrown intracorporally. For internally tied interrupted sutures, a 6 inch length is sufficient.

The two first sutures are placed at 5 and $70^{\prime}$ clock, going inside-out on the urethra and outside-in on the bladder neck. These two sutures are tied inside the urethral lumen. A metal sound with a depressed tip helps guide the needle into the urethra and place the sutures, which glide into the lumen as the sound is retracted (Figure 17).

Four other sutures are symmetrically placed, at 4 and 8 , then 2 and $100^{\prime}$ clock, and tied outside the lumen. As a rule, for a right-handed surgeon, the right-sided stitches go outside-in on the bladder (right hand) and inside-out on the urethra (left hand); the left-sided stitches go outsidein on the urethra and inside-out on the bladder neck. As a rule, for the righthanded surgeon, the bladder neck sutures are placed via the right para-rectus port, while the urethral sutures are placed via the left para-rectus port.

Two final stitches are placed at 1 and 11 o'clock, but not immediately tied. The
Foley catheter is inserted and its correct position is checked. Then the 2 sutures can be safely tied without any risk of piercing the catheter.

The balloon is inflated and the bladder is filled with 120 cc of salineto check thewatertightness of the anastomosis.

\section{Completing the operation:}

An endoscopic bag is passed through the 10$\mathrm{mm}$ port at McBumey's point and opened (Figure 18). The specimen is placed in the sac, and theneck of the sac is drawn closed. The string for the sac is cut. The port is removed and replaced such that the string to the sac now lies outside of the port. A mosquito clampisplaced on theabdominal end of the string.

The abdominal pressure is lowered to 5 $\mathrm{mm} \mathrm{Hg}$ to check for venous bleeding. The peritoneal incisions are left open, and a drain is passed to the pelvis through the lowermost left-sided port; the drain is sutured to the skin. The lower right 10$\mathrm{mm}$ trocar is removed, and the specimen is extracted; the abdominal muscles are

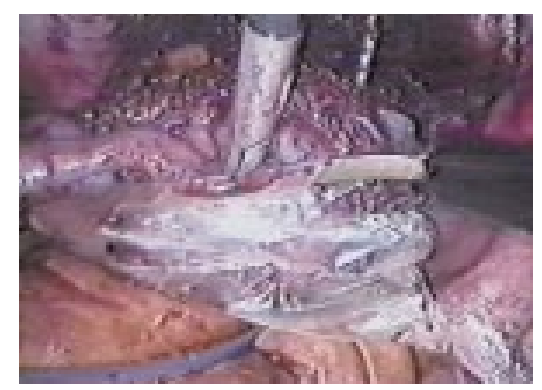

Figure 18. Specimen extraction: The specimen is placed in an extraction sac, as shown here. It is then extracted as the trocar is withdrawn. split to deliver the specimen in its sac. The remaining instruments and trocars are removed, and the incisions are closed. Various needles allow the surgeon to place sutures through the entire thickness of the abdominal wall, to reduce the risk of wound bleeding. The wounds are dressed.

\section{Postoperative care:}

Recovery is the same as for open surgery, but quicker. Indeed, often no parenteral analgesia is requested by the patients. Lowmolecular weight heparin (3,500 units) is given daily for two weeks after surgery. No additional antibiotics are given beyond the initial "on-call" dose. The drain and Foley catheter are removed on the third day (cystogram is not necessary if the anastomosis was correctly formed), and the patientisdischarged. In France, patients do not like to go homewith a catheterand thus defer their departure until the catheter has been removed. Given that most patients do not even require opiate analgesia postoperatively, in many cases they can be discharged in even less time. 
C ONCLUSION

What was first tried and abandoned in 1992 now is a fait accompli - the laparoscopic, retropubic, radical prostatectomy is a safe, feasible, standardized, reproducible, and teachable procedure. While long-term and directly comparative data are not yet available, early experience with the laparoscopic technique detailed here isfavorable. If the laparoscopic procedure is shown to be equivalent or better than the open procedure, it may replace the open, retropubic, radical prostatectomy.
REFERENCES

1. Schuessler WW, Kavoussi LR, Clayman RV, Vancaillie TH: Laparoscopic radical prostatectomy: initial case report. J Urol 147:246A, 1992

2. Price DT, Chari RS, Neighbors JD Jr, Eubanks S, Schuessler WW, Preminger GM: Laparoscopic radical prostatectomy in the canine model. J Laparoendoscop Surg 6:405-412, 1996

3. RaboyA, Ferzli G, AlbertP: Initial experience with extraperitoneal endoscopic radical prostatectomy. Urology 50:849-854, 1997

4. Schuessler WW, Schulam PG, Clayman RV, Kavoussi LR: Laparoscopic radical prostatectomy: initial short-term experience. Urology 50:854-858, 1997

5. Guillonneau B, Vallancien G: Laparoscopic radical prostatectomy: the Montsouris experience. J Urol 163:418-422, 2000

6. Krongrad A: Prostateanatomy. In: Lepor H(Ed), Prostatic Diseases, WB Saunders, Philadelphia, PA, 2000

7. Steiner MS: Continence preserving anatomic radical retropubic prostatectomy. Urology 55:427-435, 2000

T H A N K S

The authors are grateful to Ralph V. Clayman, M.D. and Chandru Sundaram, M.D. for their expert input during the preparation of the monograph. 
C O N TR I B U TING A U THORS

Guy Vallancien, M.D.

Institut Mutualiste Montsouris

42, boulevard JOURDAN

75014 PARIS, FRANCE

tel: (331) 56616625

email: guy-vallancien@imm.fr
Arnon Krongrad, M.D.

The Prostate Cancer Project, Inc.

Stein Gerontological Institute

GeniatricResearch Education \&Clinical Center

Mailing Address:

4701 N. Meridian Ave, \#300

Miami Beach, FL 33140 USA

tel: (001) 305-604-2915

email: krongrad@aol.com
Bertrand Guillonneau, M.D.

Institut Mutualiste Montsouris 42, boulevard JOURDAN

75014 PARIS, FRANCE

tel: (331) 56616625

email: bguillonneau@imm.fr

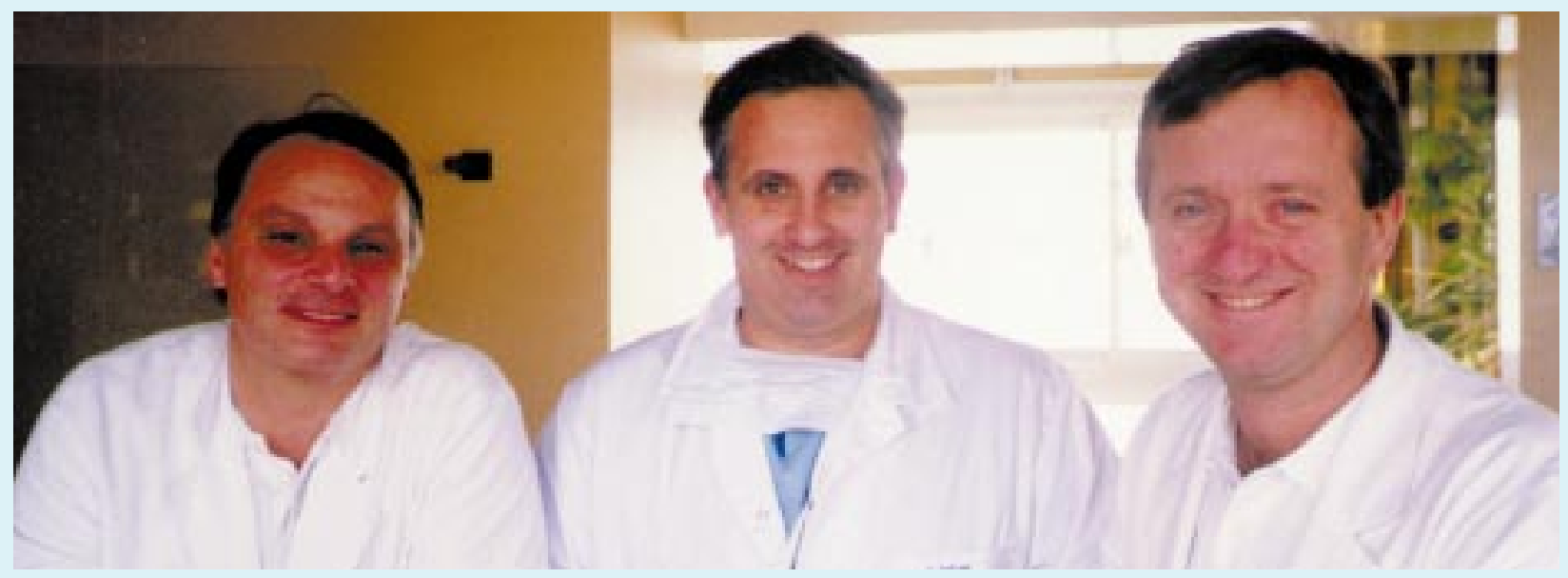

Authors (L to R): Guy Vallancien, M.D.; Arnon Krongrad, M.D.; Bertrand Guillonneau, M.D. 


\section{Provided by}

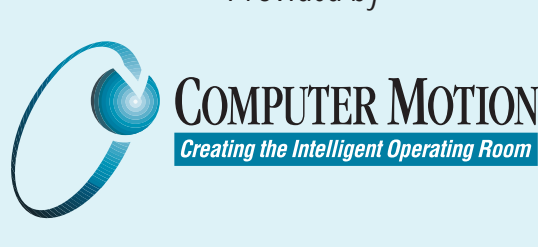

CORPORATE HEADQUARTERS:

130-B CREMONA DRIVE, SANTA BARBARA, CA 93117 USA TELEPHONE: 805-968-9600 FACSIMILE: 805-685-9277

COMPUTER MOTION EUROPE: 67091 STRASBOURG CEDEX-FRANCE

TELEPHONE: +33 388119039 FACSIMILE: +33 388119040

WWW.COMPUTERMOTION.COM 